\title{
Chidamide, a histone deacetylase inhibitor, functions as a tumor inhibitor by modulating the ratio of Bax/Bcl-2 and P21 in pancreatic cancer
}

\author{
$\mathrm{BIN} \mathrm{ZHAO}^{1}$ and TIANLIN HE ${ }^{2}$ \\ ${ }^{1}$ Department of General Surgery, Shanghai Seventh People's Hospital, Shanghai 200137; \\ ${ }^{2}$ Department of General Surgery, Changhai Hospital, Shanghai 200433, P.R. China
}

Received August 14, 2014; Accepted September 22, 2014

DOI: $10.3892 /$ or.2014.3595

\begin{abstract}
Chidamide is a newly designed histone deacetylase (HDAC) inhibitor that has been applied in clinical trials. This study aimed to test the effect of Chidamide on proliferation and apoptosis in pancreatic cancer cell lines and in vivo tumors, as well as to determine the underlying mechanism. The PaTu8988 pancreatic tumor cell line either in culture or inoculated in nude mice were used to evaluate the antitumor characteristics of Chidamide. Proliferation and apoptosis of cultured PaTu8988 cells were examined by CCK-8 assay and Annexin V-FITC/PI double staining assay, respectively. Alterations in protein expression, including Caspase-3, Bcl-2-like protein 4 (Bax), B-cell lymphoma 2 (Bcl-2) and p21, were tested by western blot analysis. The mRNA of different HDACs was examined by quantitative polymerase chain reaction (qPCR) experiments. Chidamide suppressed cell proliferation and induced early apoptosis of pancreatic tumor cells in a dose-dependent manner after $48 \mathrm{~h}$ of treatment. Similarly, the in vivo study using pancreatic tumor murine model showed that Chidamide administration significantly inhibited the growth of pancreatic tumor and induced tumor cell apoptosis. The in vitro and in vivo studies found that Chidamide treatment significantly decreased the expression of type I HDACs, uncleaved Caspase-3 and p21 and increased the ratio of $\mathrm{Bax} / \mathrm{Bcl}-2$ expression. The results from the in vitro and in vivo studies suggested Chidamide might suppress the proliferation of pancreatic tumor cells by downregulating the expression of type I HDACs and p21, and promoting mitochondrial apoptosis pathway-dependent cell apoptosis in a dose-dependent manner. The study provided more evidence for clinical administration of Chidamide
\end{abstract}

Correspondence to: Dr Tianlin He, Department of General Surgery, Changhai Hospital, 168 Changhai Road, Yangpu District, Shanghai 200433, P.R. China

E-mail: skyrainhe@163.com

Key words: chidamide, pancreatic cancer, apoptosis, histone deacetylase (HDAC) that targets pancreatic tumor cells and identified potential molecular targets for the development of potent anticancer drugs.

\section{Introduction}

Pancreatic cancer is a lethal and intractable malignancy $(1,2)$. Substantial advances have been made in understanding the biology of pancreatic cancer, as indicated by a number of risk factors that have been identified, including tobacco exposure, obesity, inherited susceptibility, persistent diabetes and pancreatitis and heavy alcohol consumption (3). Despite noteworthy developments in the treatment of pancreatic cancer, surgical resection is currently the only possible strategy of cure. Findings of recent studies have shown that $20 \%$ of the patients with pancreatic cancer is suitable for surgery, but only $5 \%$ of those patients have a 5-year survival $(4,5)$. Pancreatic cancer is not sensitive to treatment with most chemotherapeutic agents, although chemoradiation and chemotherapy are the major therapies employed for pancreatic cancer $(2,6,7)$. Thus, the poor prognosis of pancreatic cancer makes the identification of novel chemotherapeutic drugs with high efficacy and minimal side-effect imperative.

Histone deacetylase (HDAC) inhibitors are cytostatic agents that induce differentiation and apoptosis of tumor cells and have emerged as a promising new class of anticancer drugs. Chidamide, with the brand name Epidaza, is a newly designed and synthesized HDAC inhibitor, and currently studied in multiple clinical trials as a single agent or in combination with other agents for the treatment of various hematological and solid cancers (8-10). Recent findings from preclinical studies have identified that Chidamide was involved in the inhibition of proliferation and progression of various types of tumor (10-12). Recent investigations have focused on the anticancer effect-related molecular mechanisms of Chidamide in colon cancer, pancreatic cancer and leukaemia (8,13-15). For instance, Liu et al showed that Chidamide promoted apoptosis and led to cell arrest by increasing the acetylation levels of histone $\mathrm{H} 3$ and suppressing the phosphoinositide 3-kinase/Akt and MAPK/Ras signaling pathways (8). Gong et al suggested that Chidamide treatment resulted in G1 arrest at a low concentration and induced differentiation at moderate concentrations 
in human leukaemia cell lines (14). Although in a recent study Qiao et al assessed the anticancer activity of Chidamide in combination with gemcitabine in pancreatic cancer cells (16), in vivo studies are required to validate the result of the in vitro study. Investigations of the underlying molecular mechanism of the effect of Chidamide on pancreatic cancer should be conducted.

In the present study, we examined the anticancer effect of Chidamide in vitro and in vivo. The results showed that Chidamide suppressed the proliferation of pancreatic cancer cells and induced cell apoptosis in a dose-dependent manner. The in vivo study confirmed the significant inhibitory effect of Chidamide administration on pancreatic tumor growth. The results also showed that Chidamide treatment markedly decreased the expression of HDACs and p21 and promoted mitochondrial apoptosis pathway-dependent cell apoptosis by regulating the ratio of Bcl-2-like protein 4 (Bax), B-cell lymphoma 2 (Bcl-2) and uncleaved Caspase-3.

\section{Materials and methods}

Cell line and cell culture. Chidamide was purchased from Chipscreen Biosciences (Shenzhen, China) and was dissolved in dimethyl sulfoxide (DMSO) as a stock solution. The human PaTu8988 pancreatic cancer cell line was purchased from the cell bank of Shanghai, Chinese Academy of Sciences. PaTu8988 cells were cultured in Dulbecco's modified Eagle's medium [including $10 \%$ fetal bovine serum albumin, penicillin $(100 \mathrm{U} / \mathrm{ml})$ and streptomycin $(100 \mathrm{U} / \mathrm{ml})]$. The cell lines were cultured in a $37^{\circ} \mathrm{C}$ incubator with $5 \% \mathrm{CO}_{2}$.

Animals. Thirty BALB/c nude mice at the age of 5 weeks (18-22 g) were provided by the Animal Center of the Chinese Academy of Sciences. The mice were housed in an animal facility under standard laboratory conditions at a constant room temperature of $25 \pm 1^{\circ} \mathrm{C}$ with a humidity of $40-60 \%$. The animals were provided with free access to food and water under a $12 \mathrm{~h}$ dark/light cycle. Experiments were approved by the animal control committee of Shanghai Hospital, Shanghai, China.

Apoptosis assay. Cell apoptosis was quantified using the Annexin V-FITC/PI double staining kit according to the manufacturer's instructions (BioVision, Mountain View, CA, USA). PaTu8988 cells were randomized into 4 groups and incubated in the absence or presence of concentrations of $0,1.25,2.5$ and $5 \mu \mathrm{M}$ ) of Chidamide for $48 \mathrm{~h}$. Following washing, the cells were consecutively stained with Annexin-V-FITC and propidium iodide. Stained samples were analyzed by flow cytometry (FACSCalibur; BD Biosciences, Franklin Lakes, NJ, USA).

Cell proliferation assay. Proliferation of the PaTu8988 cells was evaluated using CCK-8 (Biyuntian Biotechnology, Jiangsu, China) assay according to the manufacturer's instructions. PaTu8988 cells were randomly into 4 groups and incubated in the absence or presence of concentrations of $0,1.25,2.5$ and $5 \mu \mathrm{M}$ ) of Chidamide for $48 \mathrm{~h}$. Subsequently, $10 \mu \mathrm{l}$ CCK-8 was added in each well and incubated for $2 \mathrm{~h}$. The optical density of each well was then measured with a microplate reader (Bio-Tek Co., Bedfordshire, UK) at $450 \mathrm{~nm}$. The cell survival rate was
Table I. Primers used for quantification measurements of mRNA expression.

\begin{tabular}{ll}
\hline Gene & \multicolumn{1}{c}{ Primers } \\
\hline HDAC1 & F: 5'-ACCGGGCAACGTTACGAAT-3' \\
& R: 5'-CTATCAAAGGACACGCCAAGTG-3' \\
HDAC2 & F: 5'-TCATTGGAAAATTGACAGCATAGT-3' \\
& R: 5'-CATGGTGATGGTGTTGAAGAAG-3' \\
HDAC3 & F: 5'-TTGAGTTCTGCTCGCGTTACA-3' \\
& R:5'-CCCAGTTAATGGCAATATCACAGAT-3' \\
GAPDH & F: 5'-GCTGGTCATCAACGGGAAA-3' \\
& R: 5'-ACGCCAGTAGACTCCACGACA-3' \\
\hline
\end{tabular}

F, forward; R, reverse; HDAC, histone deacetylase.

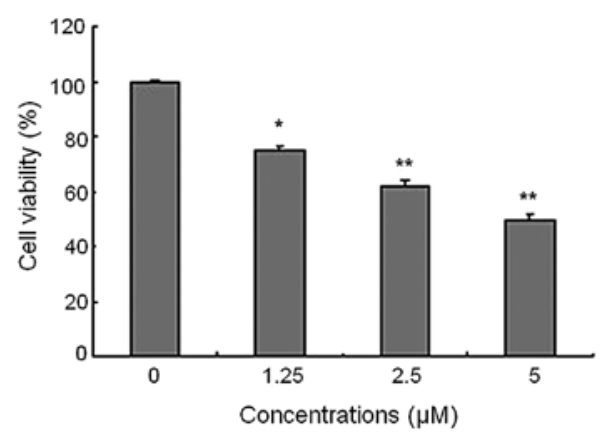

Figure 1. Chidamide inhibits the proliferation of pancreatic tumor cells in a dose-dependent manner. PaTu8988 cells were treated for $48 \mathrm{~h}$ with different concentrations $(0,1.25,2.5$ and $5 \mu \mathrm{M})$ of Chidamide. $\left({ }^{*} \mathrm{P}<0.05 ;{ }^{* *} \mathrm{P}<0.01\right.$ versus the control, one-way ANOVA).

calculated using the formula: Cell survival rate $(\%)=1-\left(\mathrm{OD}_{\mathrm{ctr}^{-}}\right.$ $\left.\mathrm{OD}_{\text {sample }}\right) / \mathrm{OD}_{\text {ctrl }} \times 100 \%$.

In vivo experiment. PaTu8988 cells $\left(2 \times 10^{7}\right)$ were suspended in phosphate-buffered saline (PBS) and subcutaneously injected into the right flank of each 5-week-old BALB/c nude mouse to establish the pancreatic tumor murine model. Mice were selected for subsequent experiments when the diameter of the pancreatic tumors ranged from 0.5 to $1 \mathrm{~cm}$. Twenty-four mice with approximately uniform tumor size were randomly divided into the control [intraperitoneal (i.p.) PBS], low Chidamide (i.p. $12.5 \mathrm{mg} / \mathrm{kg} /$ day chidamide) and high chidamide (i.p. $50 \mathrm{mg} / \mathrm{kg} /$ day chidamide) groups. PBS (vehicle) or Chidamide were administered consecutively for 21 days.

During the 21 days, mice body weight and tumor volume were measured at intervals of 3 days. Tumor volume was calculated using the formula: $\mathrm{V}=1 / 2 \mathrm{x} \mathrm{ab}^{2}$, where $\mathrm{a}$ is the maximum and $b$ the minimum length of tumor. The mice were sacrificed by cervical dislocation after 21 days. Tumor was isolated as a whole from each mouse to measure tumor weight. Tumor sections were prepared and fixed in formalin for subsequent experiments.

Hematoxylin and eosin (H\&E) staining. Tumor sections $(5 \mu \mathrm{m})$ were stained with hematoxylin for $10 \mathrm{~min}$, followed by $1 \mathrm{sec}$ 

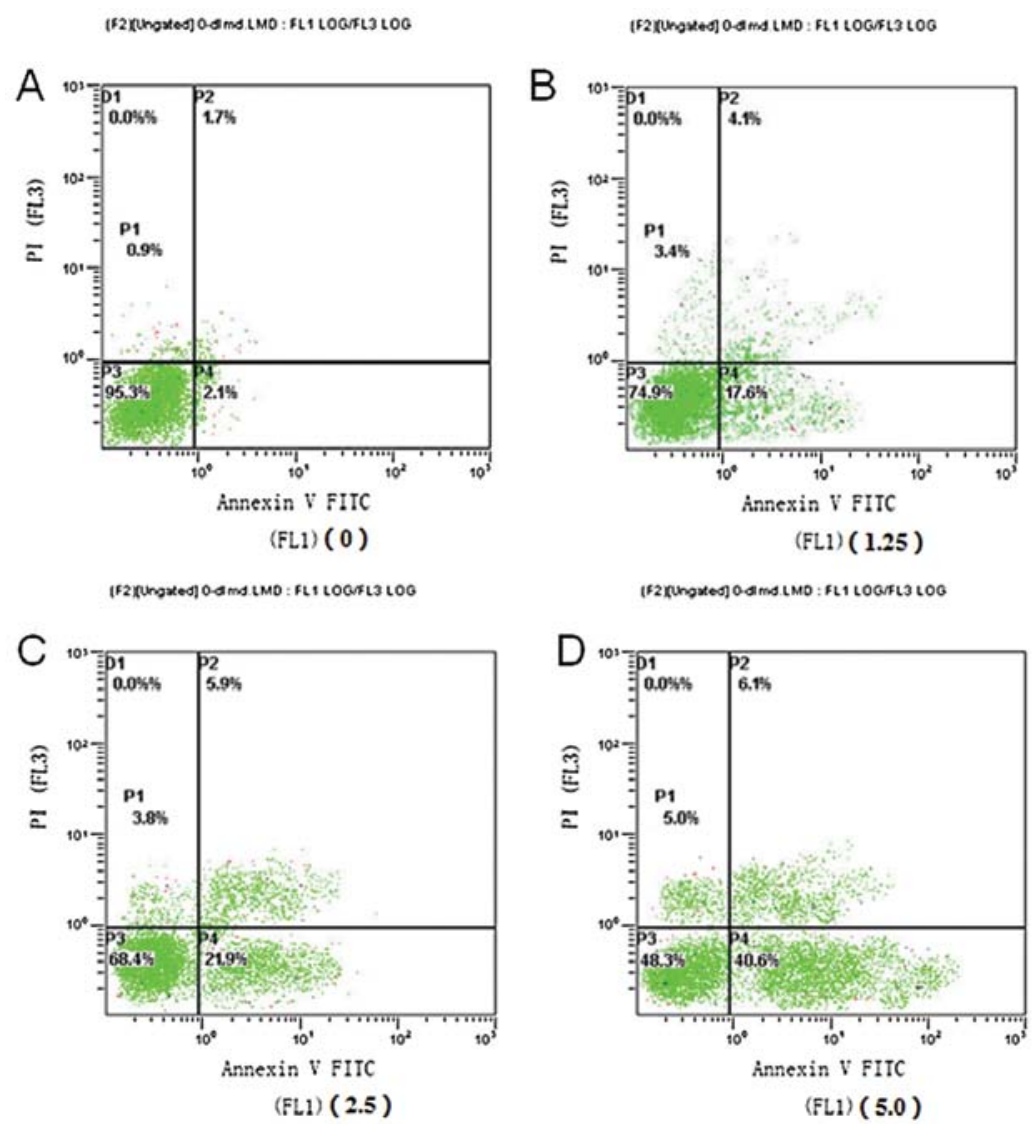

Figure 2. Chidamide induces apoptosis of human pancreatic tumor cells in a dose-dependent manner. The apoptosis rate of cells cultured in the (A) control, (B) $1.25 \mu \mathrm{M}$ Chidamide, (C) $2.5 \mu \mathrm{M}$ Chidamide and (D) $5 \mu \mathrm{M}$ Chidamide. P1, autophagic cell death; $\mathrm{P} 2$, late apoptotic cells; $\mathrm{P} 3$, normal cells; $\mathrm{P} 4$, early apoptotic cells .

differentiation in $1 \%$ hydrochloric acid and 20-30 sec staining with eosin solution, sequentially. The sections were mounted for pathological observations.

Quantitative polymerase chain reaction ( $q P C R)$. Total RNA of the PaTu8988 pancreatic cancer cell line and mice tumor tissue was extracted using TRIzol reagent (Invitrogen Life Technologies, Carlsbad, CA, USA). Total mRNA was reverse transcribed using the reverse transcription kit (Promega, Madison, WI, USA). Primers (Shenggong Bioengineering Co., Shanghai, China) used for quantification measurements are shown in Table I. GAPDH was used as an internal standard.

Total cDNA was used as a template for qPCR with the standard protocol using $2 \mu \mathrm{l}$ cDNA template, $12.5 \mu \mathrm{l}$ SYBR-Green Premix Ex Taq ${ }^{\mathrm{TM}}, 1 \mu 1$ forward primer, $1 \mu 1$ reverse primer and $8.5 \mu \mathrm{l} \mathrm{dH_{2 }} \mathrm{O}$. The thermal cycling conditions for PCR included 40 cycles of denaturation at $95^{\circ} \mathrm{C}$ for $15 \mathrm{sec}$, annealing at $55^{\circ} \mathrm{C}$ for $15 \mathrm{sec}$ and extension at $72^{\circ} \mathrm{C}$ for $15 \mathrm{sec}$. The results were analyzed using $2^{-\Delta \Delta \mathrm{Ct}}$, in which $\Delta \mathrm{Ct}=\mathrm{Ct}$ (target gene) $-\mathrm{Ct}$ (internal reference), $\Delta \Delta \mathrm{Ct}=\Delta \mathrm{Ct}$ (sample) - $\Delta \mathrm{Ct}$ (control).

Western blot analysis. The expression of Bax, Bcl-2, Caspase-3 and p21 was examined by western blot analysis according to the standard protocol. Primary antibodies used for Bcl-2 and Bax at a dilution of 1:200 were purchased from Santa Cruz Biotechnology, Inc. (CA, USA). Primary antibodies used for Caspase-3 and p21 at a dilution of 1:200 were purchased from Cell Signaling Technology (Danvers, MA, USA). Band intensities were measured using image analysis software and expressed as ratios to $\beta$-actin (internal reference).

Statistical analysis. The results were analyzed using one-way ANOVA with post-test of the Bonferroni test in SPSS13.0 software. $\mathrm{P}<0.05$ was considered to indicate a significant difference.

\section{Results}

Chidamide inhibits pancreatic tumor cell proliferation.CCK-8 assay was used to examine the proliferation of PaTu8988 cells in response to Chidamide treatment at concentrations of $0,1.25,2.5$ and $5 \mu \mathrm{M}$. As shown in Fig. 1, Chidamide caused a significant concentration-dependent inhibitory effect on cell proliferation in comparison to the vehicle-treated cells $(\mathrm{P}<0.05)$. The maximal inhibitory effect was reached at $5 \mu \mathrm{M}$.

Chidamide induces cell apoptosis in vitro and in vivo. Flow cytometry was performed to examine the apoptotic rate of PaTu8988 cells $48 \mathrm{~h}$ after chidamide administration using the Annexin V-FITC/PI double staining method (Fig. 2). Notably, cell exposure to chidamide resulted in enhanced accumulation of autophagic, early and late apoptotic cells in a dose-dependent manner, especially for the early apoptotic cells.

The histological morphology of the pancreatic tumors in nude mice was examined using H\&E staining. In the control mice administered with vehicle, a compact mass of tumor cells 
Table II. Measurement of variables relating to pancreatic tumors in mice prior to and following Chidamide treatment.

\begin{tabular}{|c|c|c|c|c|c|c|}
\hline \multirow[b]{2}{*}{ Group } & \multicolumn{2}{|c|}{ Body weight (g) } & \multicolumn{2}{|c|}{ Tumor volume $\left(\mathrm{mm}^{3}\right)$} & \multirow[b]{2}{*}{ Tumor weight (mg) } & \multirow[b]{2}{*}{ Suppression rate $(\%)$} \\
\hline & Before & After & Before & After & & \\
\hline $\begin{array}{l}\text { Control } \\
\text { (PBS) }\end{array}$ & $21.54 \pm 2.24$ & $20.58 \pm 2.88$ & $166.97 \pm 42.01$ & $485.42 \pm 71.87^{\mathrm{b}}$ & $371.24 \pm 56.21$ & 0 \\
\hline $\begin{array}{l}\text { Chidamide } \\
(12.5 \mathrm{mg} / \mathrm{kg})\end{array}$ & $20.85 \pm 2.73$ & $24.03 \pm 3.14$ & $155.98 \pm 63.27$ & $213.88 \pm 49.21^{\mathrm{ac}}$ & $197.84 \pm 49.87^{\mathrm{e}}$ & $46.71 \pm 11.28$ \\
\hline $\begin{array}{l}\text { Chidamide } \\
(50 \mathrm{mg} / \mathrm{kg})\end{array}$ & $22.05 \pm 225$ & $22.37 \pm 3.01$ & $168.09 \pm 52.84$ & $187.83 \pm 31.56^{\mathrm{ad}}$ & $146.88 \pm 34.14^{\mathrm{e}}$ & $60.44 \pm 39.26$ \\
\hline
\end{tabular}

${ }^{\mathrm{a}} \mathrm{P}<0.05$; ${ }^{\mathrm{b}} \mathrm{P}<0.01$, tumor volume, as compared to before drug application using one-way ANOVA; ${ }^{\mathrm{c}} \mathrm{P}<0.05$; ${ }^{\mathrm{d}} \mathrm{P}<0.01$, tumor volume, as compared to the control group using one-way ANOVA; ${ }^{\mathrm{P}}<0.05$, tumor weight, as compared to the control group, using one-way ANOVA. Before, prior to Chidamide treatment; after, following Chidamide treatment.
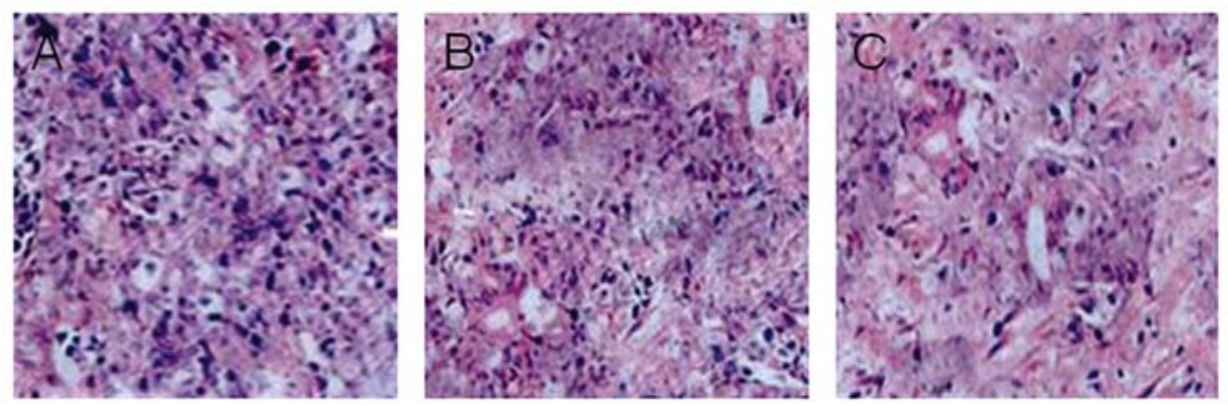

Figure 3. Hematoxylin and eosin (H\&E) staining of tumor tissue from nude mice. H\&E staining of pancreatic tumor in (A) control mice, (B) in mice treated with $12.5 \mathrm{mg} / \mathrm{kg}$ Chidamide and (C) mice treated with $50 \mathrm{mg} / \mathrm{kg}$ Chidamide.

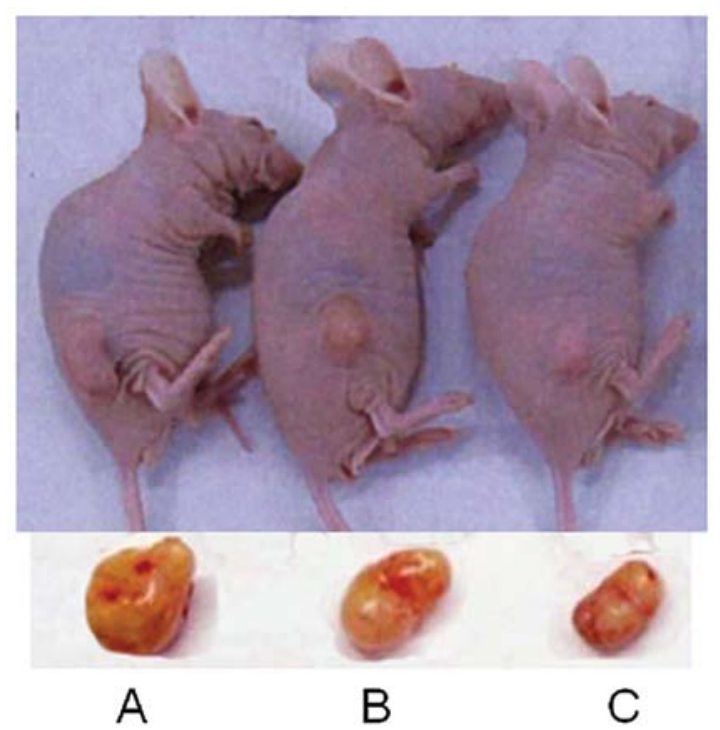

Figure 4. Chidamide inhibits the growth of pancreatic tumors in nude mice. The nude mouse (upper panel) and pancreatic tumor (bottom panel) in (A) the control condition, (B) after treatment of $12.5 \mathrm{mg} / \mathrm{kg}$ Chidamide and (C) after treatment of $50 \mathrm{mg} / \mathrm{kg}$ Chidamide in pancreatic tumor murine models.

was observed with significant nuclear fission and mitosis. By contrast, tumors treated with 12.5 or $50 \mathrm{mg} / \mathrm{kg}$ chidamide exhibited increased cell apoptosis with less cell proliferation (Fig. 3A-C).
Chidamide inhibits the growth of pancreatic tumors in nude mice. To confirm the effect of Chidamide in vivo, pancreatic tumor nude murine models were established by subcutaneous injection of PaTu8988 cells. On the second day after injection, a rice grain-size tumor was palpable. On day 7 , the tumor size was $\sim 100 \mathrm{~mm}^{3}$ and the tumor formation rate was $100 \%$. Twenty-four mice with comparable-size tumors were selected for subsequent experiments. The body weight of mice in the control group was decreased, while the body weight of the mice in the Chidamide-treated group was increased after 21 days of administration. Increases in tumor volume and tumor weight were also significantly arrested by Chidamide in a dose-dependent manner. Representative examples of pancreatic tumor from mice are shown in Fig. 4 for each group. The tumors in Chidamide-treated mice grew at a significantly reduced rate compared to those in the control group $(\mathrm{P}<0.05)$ (Table II).

Effects of Chidamide treatment on the mRNA expression level of HDACs. The expression levels of different members of HDACs class I (HDAC1, HDAC2 and HDAC3) were examined in Chidamide- and vehicle-untreated PaTu8988 cells and pancreatic tumor mice. Chidamide exposure significantly decreased the mRNA level of HDAC1, HDAC2 and HDAC3 (Fig. 5A) in a dose-dependent manner in PaTu8988 cells $(\mathrm{P}<0.05)$. Similarly, as shown in Fig. $5 \mathrm{~B}$, in the pancreatic tumor nude mice, Chidamide exerted a dose-dependent 
A
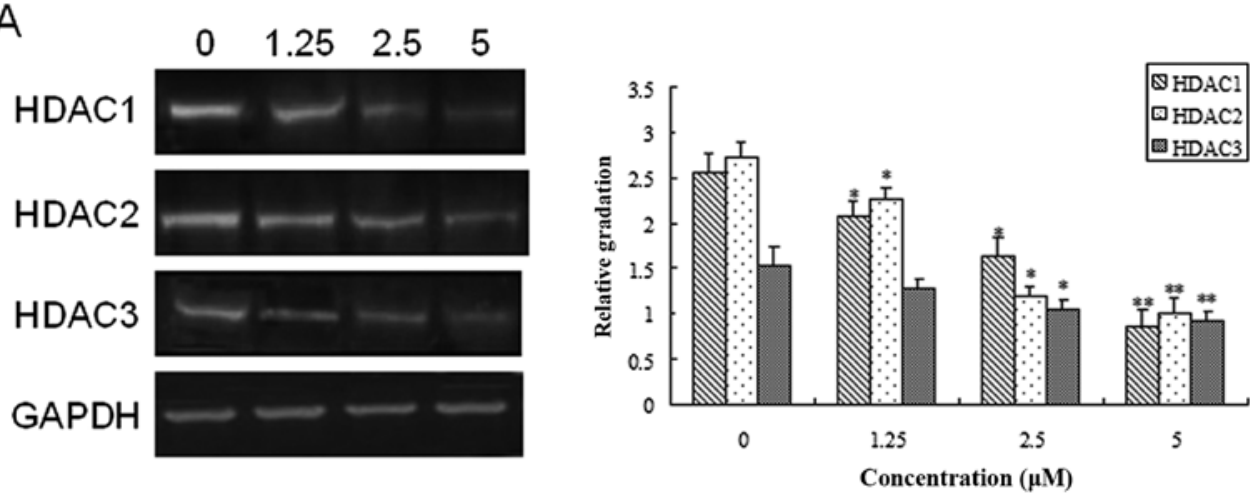

B
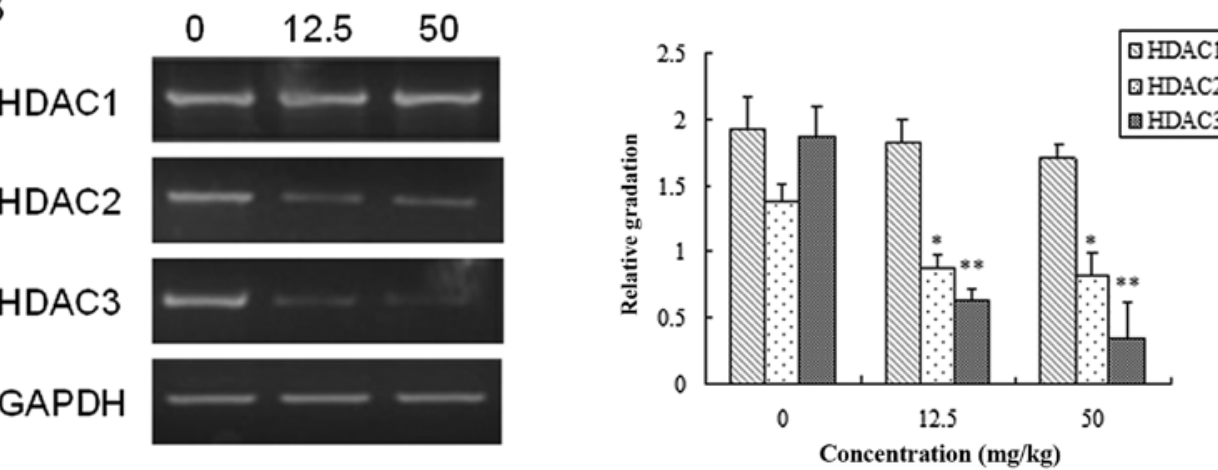

Figure 5. Chidamide suppresses the expression of histone deacetylase (HDAC) 1, HDAC2 and HDAC3 in PaTu8988 cells and mice tumor models. (A) PaTu8988 cells were incubated in the absence or presence of different concentrations $(0,1.2,2.5$ and $5 \mu \mathrm{M})$ of Chidamide. Left panel, representative blot of mRNAs from PaTu8988 cells; right panel, quantitative analysis of mRNA expression as indicated. (B) Murine tumor models were administered with PBS, $12.5 \mathrm{or} 50 \mathrm{mg} / \mathrm{kg}$ Chidamide. Left panel, representative blot of mRNAs from murine tumor models; right panel, quantitative analysis of mRNAs as indicated. $\left({ }^{*} \mathrm{P}<0.05{ }^{* * *} \mathrm{P}<0.01\right.$ versus the control, one-way ANOVA). GAPDH served as the internal control.

inhibitory effect on the mRNA levels of HDAC2 and HDAC3 $(\mathrm{P}<0.05)$, but had little impact on HDAC1 expression in pancreatic tumor nude mice at different doses $(12.5$ and $50 \mathrm{mg} / \mathrm{kg})$. Taken together, these results suggested that Chidamide may arrest the growth of pancreatic tumor through inhibition of the expression of HDACs, which may be involved in the underlying mechanism for the therapeutic effect of Chidamide on pancreatic cancer.

Chidamide decreases the expression level of Bcl-2 and Caspase-3 and increases the expression level of Bax and 21. To gain some insight into the molecular mechanism of the tumor-inhibiting effect of chidamide, the protein expression levels of Bcl-2, Bax, Caspase-3 and p21 were further investigated in vitro and in vivo. In PaTu8988 cells, chidamide treatment significantly enhanced the expression of Bax, but suppressed the expression of Bcl-2 and uncleaved Caspase-3 (Fig. 6A) in a dose-dependent manner $(\mathrm{P}<0.05)$. Since Caspase- 3 is the downstream signal of the mitochondrial apoptotic pathway (3), these data suggested that application of Chidamide might promote cell apoptosis through the mitochondrial apoptotic pathway.

Consistent with the result of the in vitro experiments, in the pancreatic tumor nude mice Chidamide administration led to a significant increase in the expression of Bax and p21 and a decrease in the expression of Bcl-2 (Fig. 6B) $(\mathrm{P}<0.05)$. The effect of Chidamide was dependent on the administration dose. The results from the in vitro and in vivo studies provide convincing evidence for the view that Chidamide may affect the apoptosis and proliferation of pancreatic tumor cells by modulating the mitochondrial apoptotic pathway and the expression of $\mathrm{p} 21$, respectively.

\section{Discussion}

Pancreatic cancer is an excruciating gastrointestinal tumor, characterized by poor prognosis and an exceedingly high death rate (17). Currently, there is a shortage of potent chemotherapeutic agents with few side effects that may be used to treat the disease and prolong patient survival. In the present study, we emphasized the effect of Chidamide on inhibiting the proliferation of pancreatic tumor cell in vitro and in vivo. We found that administration of Chidamide suppressed the proliferation of pancreatic tumor cells and induced cell apoptosis in a dose-dependent manner.

Chidamide functions as a HDAC inhibitor which is newly designed and synthesized in China (8) and may specifically suppress the level of type I HDACs. Increasing findings, from in vitro studies of tumor cell lines to in vitro studies of animal tumor models, support that Chidamide is a potential therapeutic drug in the treatment of a variety of cancers such as hepatocellular carcinoma, lymphoma cancer and bladder tumor $(12,18,19)$. Despite substantial progress in understanding the effect of Chidamide on cancer, the underlying 
A
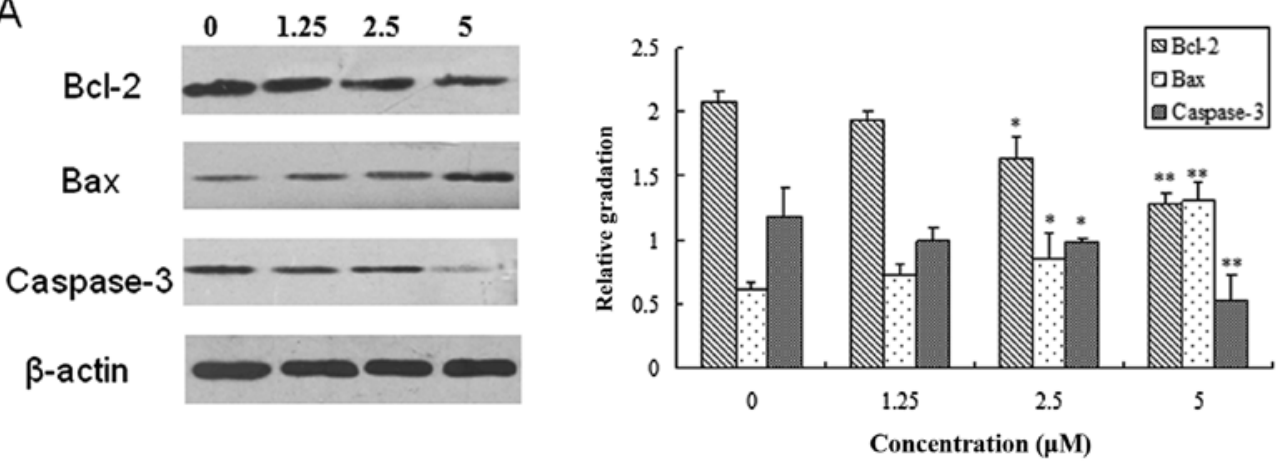

B
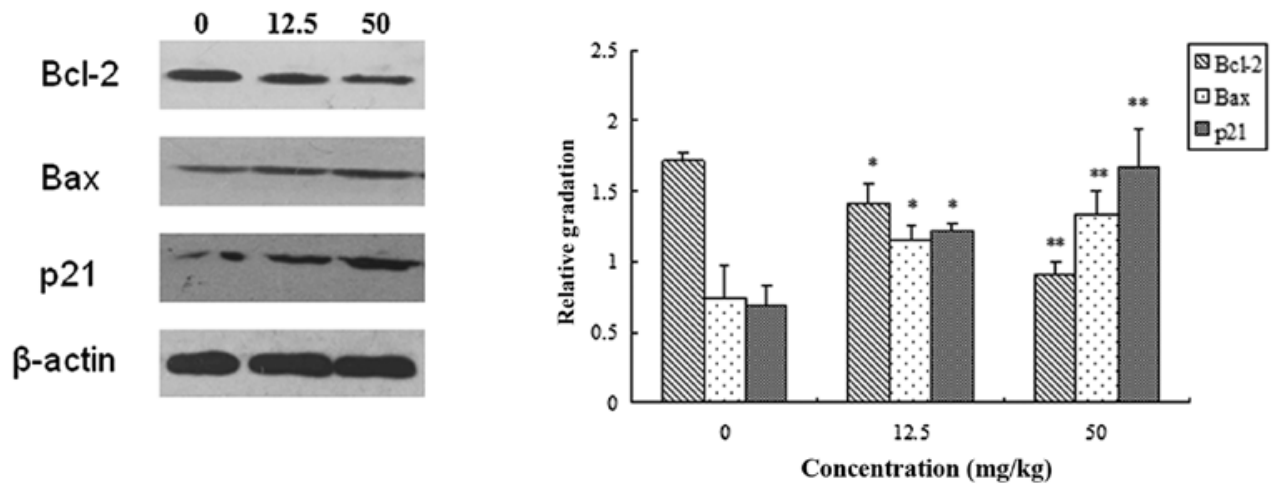

Figure 6. Chidamide modulated the expression of B-cell lymphoma 2 (Bcl-2), Bax, Caspase-3 and p21 in pancreatic cancer. (A) PaTu8988 cells were incubated in the absence or presence of different concentrations $(0,1.25,2.5$ and $5 \mu \mathrm{M})$ of Chidamide. Left panel, representative immunoblot of proteins; right panel, quantitative analysis of expression of proteins as indicated. (B) Murine tumor models were administered with PBS, $12.5 \mathrm{mg} / \mathrm{kg}$ or $50 \mathrm{mg} / \mathrm{kg}$ Chidamide. Left panel, representative immune-blot of proteins as indicated; Right, quantitative analysis of expression of proteins as indicated. $\left({ }^{*} \mathrm{P}<0.05\right.$; ${ }^{* *} \mathrm{P}<0.01$, versus the control using one-way ANOVA).

molecular mechanism remains to be determined. Therefore, we investigated the therapeutic effect of chidamide on the treatment of pancreatic cancer and discussed possible mechanism underlying this effect.

First, we found that Chidamide can suppress the proliferation of pancreatic tumor cells in a dose-dependent manner via CKK-8 assay. The homeostasis of cell proliferation is regulated by cell apoptosis, thus it is of critical significance to target apoptotic proteins for the treatment of pancreatic cancer. Two major apoptosis pathways include intrinsic mitochondrial apoptosis and extrinsic death receptor pathways or endoplasmic reticulum stress pathways (20). The mitochondrial apoptosis pathway is the most important pathway by regulating the ratio of apoptotic proteins Bcl-2 and Bax. Specifically, Bcl-2 suppresses apoptosis partly by blocking efflux of cytochrome $c$ which activates downstream caspase signals, while Bax has an apoptosis-promoting effect by antagonizing Bcl-2 $(12,21)$. Therefore, the ratio of $\mathrm{Bcl}-2 / \mathrm{Bax}$ expression determines whether the apoptotic process occurs. An increased Bax/Bcl-2 ratio can activate Caspase-3 and result in cell death (22-24). In this study, the effect of Chidamide on these apoptosis-related proteins was also investigated. We found that in PaTu8988 cells, Chidamide treatment promoted the expression of Bax, but inhibited the expression of Bcl-2 and uncleaved Caspase- 3 in a dose-dependent manner. The results suggested that application of Chidamide significantly enhanced the apoptotic process by activation of Caspase- 3 which is the downstream signal of the mitochondrial apoptotic pathway. Chidamide treatment had a similar effect on the expression of Bax and Bcl-2 in in vivo experiments of pancreatic tumor nude mice. These findings suggest that the inhibitory effect of Chidamide administration on pancreatic tumor cells may be partly attributed to the activation of mitochondrial apoptosis pathways.

It has been reported that HDAC inhibitors can induce the activation of multiple signaling pathways, involving p53, p21 and $\mathrm{Rb}$ proteins that can result in cell cycle arrest and apoptosis (25). P21, also known as cyclin-dependent kinase inhibitor 1 has an important role in modulating cell cycle and mediating cell senescence. P21 plays a positive role in the suppression of tumor cell growth and proliferation (26-28). Our study results revealed that Chidamide administration inhibited pancreatic tumor growth by promoting p21 expression. HDAC is an enzyme family in which type I HDAC includes HDAC1, HDAC2, HDAC3 and HDAC8. The mRNA level of HDAC2 and HDAC3 was significantly decreased in human pancreatic cancer cells and pancreatic tumor nude mice in response to Chidamide administration. The inhibitory effect of Chidamide was positively correlated with its administration dose. Taken together, these results suggest that HDACs may participate in the growth of pancreatic tumor and Chidamide administration may suppress tumor growth through inhibition of HDAC and promotion of $\mathrm{p} 21$ expression. Thus, $\mathrm{p} 21$ is a potential candidate target for the therapeutic treatment of pancreatic cancer.

In conclusion, this study have confirmed the inhibitory effect of Chidamide on pancreatic cells by in vitro and in vivo studies and suggested that Chidamide may promote 
cell apoptosis via mitochondrial apoptosis pathway and inhibit cell growth by downregulating the expression of $\mathrm{p} 21$, thus suppressing the progression of pancreatic cancer. Our study provides strong evidence for the clinical application of Chidamide as a tumor-inhibiting agent and promoted the development of novel therapeutic strategies with high efficacy that could lead to improved treatment of the pancreatic cancer. Further studies on the combined effect of Chidamide and other chemotherapeutic agents are necessary for clinical application.

\section{Acknowledgements}

This study was sponsored by the Shanghai Pujiang Program (13PJD001).

\section{References}

1. Lennon AM, Wolfgang CL, Canto MI, et al: The early detection of pancreatic cancer: What will it take to diagnose and treat curable pancreatic neoplasia? Cancer Res 74: 3381-3389, 2014.

2. Vincent A, Herman J, Schulick R, Hruban RH and Goggins M: Pancreatic cancer. Lancet 378: 607-620, 2011.

3. Zhu L, Yuan H, Guo C, et al: Zearalenone induces apoptosis and necrosis in porcine granulosa cells via a caspase-3- and caspase-9-dependent mitochondrial signaling pathway. J Cell Physiol 227: 1814-1820, 2012.

4. Siegel R, Naishadham D and Jemal A: Cancer statistics, 2013. CA Cancer J Clin 63: 11-30, 2013.

5. Shaib Y, Davila J and El-Serag H: The epidemiology of pancreatic cancer in the United States: changes below the surface. Aliment Pharmacol Ther 24: 87-94, 2006.

6. Sultana A, Smith CT, Cunningham D, Starling N, Neoptolemos JP and Ghaneh P: Meta-analyses of chemotherapy for locally advanced and metastatic pancreatic cancer. J Clin Oncol 25: 2607-2615, 2007.

7. Burris HA III, Moore MJ, Andersen J, et al: Improvements in survival and clinical benefit with gemcitabine as first-line therapy for patients with advanced pancreas cancer: a randomized trial. J Clin Oncol 15: 2403-2413, 1997.

8. Liu L, Chen B, Qin S, et al: A novel histone deacetylase inhibitor Chidamide induces apoptosis of human colon cancer cells. Biochem Biophys Res Commun 392: 190-195, 2010.

9. Dong M, Ning ZQ, Xing PY, et al: Phase I study of chidamide (CS055/HBI-8000), a new histone deacetylase inhibitor, in patients with advanced solid tumors and lymphomas. Cancer Chemother Pharmacol 69: 1413-1422, 2012.

10. Zhou Y, Pan DS, Shan S, et al: Non-toxic dose chidamide synergistically enhances platinum-induced DNA damage responses and apoptosis in non-small-cell lung cancer cells. Biomed Pharmacother 68: 483-491, 2014.

11. Liu L, Chen B, Qin S, et al: A novel histone deacetylase inhibitor Chidamide induces apoptosis of human colon cancer cells. Biochem Biophys Res Commun 392: 190-195, 2010.

12. Wang H, Guo Y, Fu M, et al: Antitumor activity of Chidamide in hepatocellular carcinoma cell lines. Mol Med Rep 5: 1503-1508, 2012.
13. NingZQ,LiZB,Newman MJ, etal: Chidamide(CS055/HBI-8000): a new histone deacetylase inhibitor of the benzamide class with antitumor activity and the ability to enhance immune cellmediated tumor cell cytotoxicity. Cancer Chemother Pharmacol 69: 901-909, 2012.

14. Gong K, Xie J, Yi H and Li W: CS055 (Chidamide/HBI-8000), a novel histone deacetylase inhibitor, induces G1 arrest, ROSdependent apoptosis and differentiation in human leukaemia cells. Biochem J 443: 735-746, 2012.

15. Qiao Z, Ren S, Li W, et al: Chidamide, a novel histone deacetylase inhibitor, synergistically enhances gemcitabine cytotoxicity in pancreatic cancer cells. Biochem Biophys Res Commun 434: 95-101, 2013

16. Qiao Z,Ren S, Li W, et al: Chidamide, a novel histone deacetylase inhibitor, synergistically enhances gemcitabine cytotoxicity in pancreatic cancer cells. Biochem Biophys Res Commun 434: 95-101, 2013.

17. Li D, Xie K, Wolff R and Abbruzzese JL: Pancreatic cancer. Lancet 363: 1049-1057, 2004.

18. Yen MC, Weng TY, Chen YL, et al: An HDAC inhibitor enhances cancer therapeutic efficiency of RNA polymerase III promoter-driven IDO shRNA. Cancer Gene Ther 20: 351-357, 2013.

19. Ning Z-Q, Li Z-B, Newman MJ, et al: Chidamide (CS055/HBI8000): a new histone deacetylase inhibitor of the benzamide class with antitumor activity and the ability to enhance immune cell-mediated tumor cell cytotoxicity. Cancer Chemother Pharmacol 69: 901-909, 2012.

20. Fiandalo MV and Kyprianou N: Caspase control: protagonists of cancer cell apoptosis. Exp Oncol 34: 165-175, 2012.

21. Yang J, Liu X, Bhalla K, et al: Prevention of apoptosis by Bcl-2: release of cytochrome $\mathrm{c}$ from mitochondria blocked. Science 275: 1129-1132, 1997.

22. Siu WP, Pun PB, Latchoumycandane C and Boelsterli UA: Bax-mediated mitochondrial outer membrane permeabilization (MOMP), distinct from the mitochondrial permeability transition, is a key mechanism in diclofenac-induced hepatocyte injury: Multiple protective roles of cyclosporin A. Toxicol Appl Pharmacol 227: 451-461, 2008.

23. Ohtsuka T, Buchsbaum D, Oliver P, Makhija S, Kimberly R and Zhou T: Synergistic induction of tumor cell apoptosis by death receptor antibody and chemotherapy agent through JNK/p38 and mitochondrial death pathway. Oncogene 22: 2034-2044, 2003.

24. Elmore S: Apoptosis: a review of programmed cell death. Toxicol Pathol 35: 495-516, 2007.

25. Huang WJ, Liang YC, Chuang SE, et al: NBM-HD-1: A novel histone deacetylase inhibitor with anticancer activity. Evid Based Complement Alternat Med 2012: 781417, 2012.

26. Ohashi K, Nemoto T, Eishi Y, Matsuno A, Nakamura K and Hirokawa K: Expression of the cyclin dependent kinase inhibitor p21WAF1/CIP1 in oesophageal squamous cell carcinomas. Virchows Arch 430: 389-395, 1997.

27. Dai L, Liu Y, Liu J, et al: A novel cyclinE/cyclinA-CDK inhibitor targets $\mathrm{p} 27^{\mathrm{Kipl}}$ degradation, cell cycle progression and cell survival: implications in cancer therapy. Cancer Lett 333: 103-112, 2013.

28. Hoefer J, Schäfer G, Klocker H, et al: PIAS1 is increased in human prostate cancer and enhances proliferation through inhibition of p21. Am J Pathol 180: 2097-2107, 2012. 